\title{
Prokrastination als Coaching-Anliegen
}

\author{
Michaela Riediger
}

Online publiziert: 24. Oktober 2016

(C) Der/die Autor(en) 2016. Dieser Artikel ist eine Open-Access-Publikation.

Zusammenfassung Prokrastination betrifft das nicht zielgerichtete und nicht durch äußere Zwänge hervorgerufene Aufschieben intendierter Handlungen. Es tritt häufig auf und hat potenziell negative Auswirkungen sowohl auf die betroffene Person als auch auf die Organisationseinheit, in der sie tätig ist. Anliegen im Coaching können mit eigenen Prokrastinationstendenzen des Coachees ebenso wie mit denen seiner Mitarbeiter oder Kollegen im Zusammenhang stehen. Die „Temporal Motivation Theory" bietet sich in solchen Fällen als theoretischer Rahmen für Strukturierung des Coaching-Prozesses und die Auswahl geeigneter Coaching-Werkzeuge an.

Schlüsselwörter Prokrastination · Temporal Motivation Theory $\cdot$ Coaching

\section{Procrastination as a coaching topic}

Abstract Procrastination refers to non-deliberately delaying intended actions in the absence of external constraints that force one to do. It occurs frequently and can have negative consequences both for the procrastinating individual and the organizational unit in which he or she works. Procrastination tendencies of individuals themselves or of other people that they are working with can be topics that are addressed in coaching processes. The Temporal Motivation Theory provides a theoretical framework that is well suited as a guideline for structuring the coaching process in those instances and for selecting appropriate coaching tools.

Keywords procrastination · Temporal Motivation Theory $\cdot$ Coaching

Dr. M. Riediger $(\bowtie)$

Freie Universität Berlin und Max-Planck-Institut für Bildungsforschung,

Lentzeallee 94, 14195 Berlin, Deutschland

E-Mail: riediger@mpib-berlin.mpg.de 


\section{Einleitung}

So wie Duke Ellington geht es vielen Menschen. Sie schieben die Erledigung von Aufgaben so lange auf, bis die Deadline schließlich in bedrohliche Nähe rückt. Auch wenn ursprünglich ausreichend Zeit zur Verfügung stand, geraten sie so schließlich in Zeitnot. Stress, Hektik und beeinträchtigte Leistungsergebnisse sind die Folgen. In Fachkreisen wird dieses unnötige und dysfunktionale Aufschieben von intendierten Aktivitäten als „Prokrastination“ bezeichnet (lat. procrastinatio = ,Vertagung“). Im Berufsalltag von Führungskräften kann Prokrastination auf verschiedenen Ebenen zum Anliegen werden. Zum einen können die Leistungsfähigkeit, Gesundheit und das Wohlbefinden von Führungskräften unter ihrer eigenen Prokrastinationsneigung leiden. Zum anderen kann Prokrastination bei einem oder mehreren Mitarbeitern oder Kollegen mit Problemen im Zusammenhang stehen, die im Coaching thematisiert werden. Coaches sollten daher ein Grundverständnis zum Phänomen der Prokrastination haben und Beratungsoptionen für unterschiedliche Szenarien kennen.

Dieser Beitrag hat zum Ziel, die aktuelle psychologische Prokrastinationsforschung in ihrer Relevanz für den Coaching-Alltag zu beleuchten. Hierzu wird das Phänomen Prokrastination zunächst definiert und von anderen Formen des Handlungsaufschubs abgegrenzt. Auch werden die Prävalenz und mögliche Folgen von Prokrastination und somit die Relevanz von Prokrastination als Coaching-Gegenstand diskutiert. Anschließend werden Kernaussagen und unterstützende empirische Befunde der Temporal Motivation Theory (Steel 2007) zusammengefasst. Hierbei handelt es sich um ein Modell, das sich gut als theoretischer Rahmen zur Adressierung von Prokrastination im Coaching eignet. Es wird in diesem Beitrag als Orientierung für das Vorgehen beim Bearbeiten von Prokrastination als CoachingAnliegen und für die Auswahl geeigneter Coaching-Werkzeuge genutzt.

\section{Definition und Abgrenzung von anderen Formen des Handlungsaufschubs}

Wie lässt sich das Phänomen Prokrastination definieren? In der psychologischen Literatur finden sich unterschiedliche Definitionen, die zum Teil verschiedene Formen von Handlungsaufschub miteinander vermischen. Der vorliegende Beitrag orientiert sich an einer Definition, die von Katrin Klingsieck (2013) von der Universität Paderborn vorgeschlagen wurde und die sich eindeutig auf nicht-zielgerichtete und dysfunktionale Prokrastination beschränkt. Nach dieser Definition ist Prokrastination durch die folgenden sieben definitorischen Merkmale gekennzeichnet:

1. Eine intendierte Handlung,

2. die notwendig oder von persönlicher Bedeutung ist,

3. wird aufgeschoben,

4. obwohl dem Individuum bewusst ist, dass dies negative Konsequenzen hat. 
5. Dieser Aufschub erfolgt ohne externe Zwänge,

6. ist unnötig oder irrational (nicht zielgerichtet)

7. und geht mit subjektivem Unbehagen oder anderen negativen Konsequenzen einher.

Diese Definition grenzt Prokrastination von anderen Formen des Handlungsaufschubs ab. Eine solche abzugrenzende Form des Handlungsaufschubs ist beispielsweise das sogenannte Self-Handicapping. Dies bezeichnet den gezielten Aufschub von Aufgaben, um im Falle von Misserfolg eine selbstwertdienliche Erklärung zu haben (Rhodewalt und Vohs 2005). Ein Beispiel wäre, vor einem wichtigen Präsentationstermin gezielt andere, für die Präsentation irrelevante Aufgaben zu bearbeiten, obwohl dies vermeidbar wäre, um so eine möglicherweise negative Bewertung der Präsentation selbstwertdienlich mit der mangelnden Vorbereitungszeit erklären zu können (anstatt sich eingestehen zu müssen, dass die Präsentation trotz penibler Vorbereitung nicht gut ankam). In der hier verwendeten Definition von Klingsieck ist Prokrastination auch abzugrenzen von dem gezielten Aufschub von Handlungen aufgrund der Annahme, dass man unter Zeitdruck besser oder effizienter arbeiten kann - ein Phänomen, das in der Literatur als ,,active procrastination“ (Chu \& Choi 2005), ,,arousal procrastination“ (Simpson und Pychyl 2009) oder auch ,funktionaler Handlungsaufschub“ (Klingsieck 2013) bezeichnet wird. Diese beiden Formen des Handlungsaufschubs erfüllen nicht alle definitorischen Kriterien laut Klingsieck, da die Person sich hiervon einen Nutzen verspricht (für den Selbstwert oder für die Handlungseffizienz), der Aufschub also zielgerichtet erfolgt und zumindest im letzteren Fall auch nicht notwendigerweise negative Konsequenzen hat.

Der vorliegende Beitrag adressiert also nicht solche Formen des zielgerichteten Handlungsaufschubs. Auch wird es im Folgenden nicht um Formen des Handlungsaufschubs gehen, die äußeren Zwängen geschuldet sind. In bestimmten Branchen und/oder Führungskräftekreisen mag es häufig vorkommen, dass Aufgaben aufgrund von Überlastung aufgeschoben werden müssen, weil schlicht keine Zeit für deren Erledigung ist. Auch dies ist keine Prokrastination in dem hier verwendeten Sinne. Der praktisch tätige Coach sollte um diese verschiedenen Formen des Handlungsaufschubs wissen und sie durch geeignete Explorationsfragen voneinander abgrenzen können, da jeweils unterschiedliche Vorgehensweisen und Problemfelder indiziert sind.

Im Mittelpunkt dieses Beitrags steht also Prokrastination als nicht zielgerichteter Handlungsaufschub, der ohne äußere Zwänge erfolgt und die oben benannten sieben definitorischen Merkmale erfüllt.

\section{Prokrastination als Coaching-Anliegen: Prävalenz und Konsequenzen}

Die Relevanz von Prokrastination als potenzielles Anliegen im Führungskräfte-Coaching resultiert aus zwei Gründen. Prokrastination tritt zum einen häufig auf und ist zum anderen eine Verhaltenstendenz mit potenziell schwerwiegenden negativen Konsequenzen. Jeder vierte bis fünfte Erwachsene berichtet von Prokrastinationstendenzen, die so stark sind, dass er oder sie diese als belastend und problematisch 
erachtet (vgl. Rozental und Carlbring 2014). Berufliche Kontexte sind besonders prädestiniert für das Auftreten von Prokrastination, wenngleich auch andere Lebensbereiche betroffen sind. Führungskräfte werden daher recht wahrscheinlich Situationen erleben, in denen sie mit ihren eigenen und/oder den Prokrastinationstendenzen von Kollegen und Mitarbeitern konfrontiert werden. Vielfältige Studien haben zudem gezeigt, dass Prokrastination mit niedrigerem psychologischem Wohlbefinden und schlechteren Leistungen einhergeht. Besonders schwerwiegend ist auch, dass Prokrastinierer durchschnittlich krankheitsanfälliger und weniger gesund sind als weniger zur Prokrastination neigende Personen (Freund und Krause 2013).

Dies wird zum einen damit erklärt, dass Prokrastination aufgrund des Handlungsaufschubs ultimativ in Stress-Situationen mit den entsprechenden physiologischen Stress-Reaktionen resultiert. Solche Stress-Reaktionen dienen der kurzfristigen Erhöhung der Leistungs- und Handlungsfähigkeit des Organismus, führen chronifiziert jedoch zu einer Überlastung (,wear and tear“) von Herz und Kreislauf sowie von anderen Organsystemen. Zum anderen kann Prokrastination auch Gesundheitsverhaltensweisen betreffen, sich also beispielsweise in einem Aufschieben von Arztbesuchen oder gesundheitlichen Präventionsmaßnahmen äußern. Beide Prozesse chronifizierter Stress und Aufschub von gesundheitsrelevanten Handlungen - tragen zu einer erhöhten Wahrscheinlichkeit von gesundheitlichen Beeinträchtigungen bei Prokrastinierern bei (Sirois 2007). Neben diesen negativen Auswirkungen, die Prokrastination auf das Wohlbefinden, die Gesundheit und die Leistungsfähigkeit der prokrastinierenden Person selbst haben kann, sind gerade in beruflichen Kontexten auch interpersonelle und systemische Konsequenzen zu befürchten, welche die Sozialdynamik und Leistungsfähigkeit der betroffenen Organisationseinheiten beeinträchtigen können. Dies ist umso wahrscheinlicher, je stärker die prokrastinierende Person in Teamprozesse eingebunden ist, in denen andere auf ihre Arbeitsergebnisse angewiesen sind.

Zusammengenommen ist Prokrastination also ein häufiges und dysfunktionales Verhalten von hoher Relevanz in beruflichen Kontexten. Entsprechend relevant für Coaches ist also das Verständnis möglicher Einflussfaktoren auf die Prokrastinationstendenz. Denn nur ein grundlegendes Verständnis von Prokrastination liefert die Voraussetzungen für die Unterstützung effektiver Problemlösungs- und Veränderungsprozesse.

Ehe ein Erklärungsansatz von Prokrastination, der sich besonders für die Anwendung in Coaching-Kontexten eignet, vorgestellt wird, ist folgender Hinweis wichtig: Prokrastination tritt zwar häufig in der Allgemeinbevölkerung auf und ist nicht als a priori klinisch relevant zu bewerten. Dennoch sollte dem Coach bewusst sein, dass Prokrastination durchaus auch ein Symptom psychischer Erkrankung sein kann, etwa von klinisch relevanten Arbeitsstörungen, depressiven oder Zwangserkrankungen. In solchen Fällen ist ein Coaching kontraindiziert. Der Coach sollte den Ratsuchenden stattdessen ermuntern, sich therapeutische Hilfe zu suchen. 


\section{Die Temporal Motivation Theory}

Die Temporal Motivation Theory wurde von Steel und König (2006) vorgeschlagen und wird in der aktuellen nicht-klinischen Prokrastinationsforschung viel beachtet, da sie mit einer Vielzahl empirischer Befunde konsistent ist. Darüber hinaus eignet sie sich auch hervorragend als theoretischer Rahmen für den praktischen Einsatz in Coaching-Kontexten. Zum einen bietet sie dem Coach ein theoretisches Gerüst für die Exploration und anschließende Auswahl geeigneter Interventionsstrategien, wenn der Coachee eigene Prokrastinationstendenzen als Coaching-Anliegen einbringt. Auch ist sie im Zuge der Psychoedukation leicht zu vermitteln, wenn Prokrastinationstendenzen von Mitarbeitern oder Kollegen im Coaching bearbeitet werden sollen. Über solche Psychoedukation kann dem Coachee zu einem besseren Verständnis seines Anliegens verholfen werden.

\subsection{Die Kernaussagen der Theorie}

Wie der Name der Theorie schon sagt, spielt der Zeitfaktor in ihr eine zentrale Rolle, und zwar in Form eines Phänomens, das als ,temporal discounting“ bezeichnet wird. Damit ist gemeint, dass der Motivationsdruck, eine geplante Handlung tatsächlich auszuführen, umso geringer ist, je weiter das Fristende für diese Handlung (z. B. der Meilenstein in der Produktentwicklung, der Präsentationstermin in der Vorstandssitzung) entfernt ist. Kurz vor Fristende (der Deadline) steigt der Motivationsdruck jedoch stark an. Die Theorie betont zudem, dass es hier große interund intraindividuelle Unterschiede gibt. Verschiedene Personen unterscheiden sich in ihrer Anfälligkeit für das „temporal discounting“. Auch dieselbe Person zeigt dieses Phänomen nicht für alle Aufgaben im gleichen Ausmaß. Zur Erklärung dieser Unterschiede werden vier Einflussfaktoren auf den Motivationsdruck (bzw. die Prokrastinationstendenz) postuliert (vgl. Abb. 1).

Der Motivationsdruck, ein intendiertes Verhalten (z. B. eine Präsentation vorzubereiten) tatsächlich zu zeigen, ergibt sich aus dem Produkt vom (emotionalen oder rationalen) Wert der erhofften Verhaltenskonsequenz und der subjektiven Erwartung, mit dem Verhalten diese Konsequenz auch tatsächlich zu erzielen. Je höher der Wert

Abb. 1 Kernaussagen der Temporal Motivation Theory (Steel 2007)
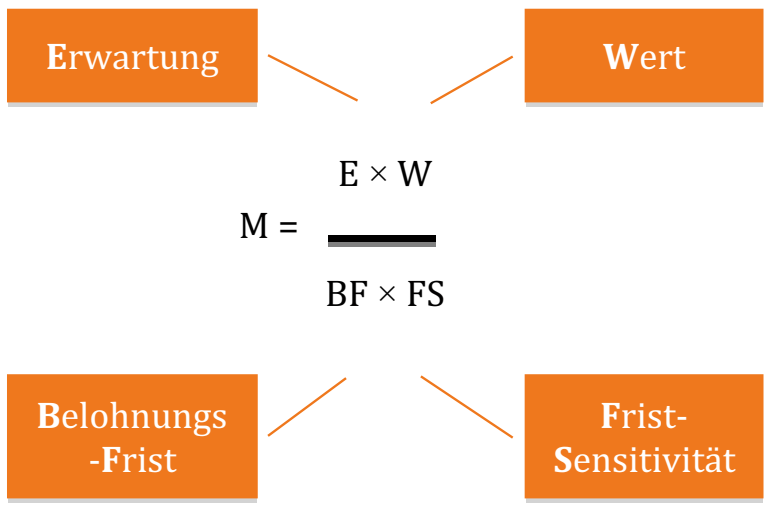
der Verhaltenskonsequenz (z. B. je wichtiger der Person eine positive Resonanz auf ihre Präsentation ist) und je höher die Erwartung, diese Verhaltenskonsequenz auch erzielen zu können (das Publikum tatsächlich positiv zu beeindrucken), desto höher ist der Motivationsdruck, das intendierte Verhalten (die Vorbereitung der Präsentation) in die Tat umzusetzen (und desto geringer ist folglich ihre Prokrastinationstendenz). Dies wird im Nenner zusätzlich kalibriert durch den verfügbaren Zeithorizont - der sogenannten Belohnungsfrist - ebenso wie durch die Sensitivität der Person, sich in ihrer Verhaltensmotivation von dieser Belohnungsfrist beeinflussen zu lassen - der sogenannten Fristsensitivität. Je mehr Zeit noch bis zum Präsentationstermin ist und je mehr die Person dazu tendiert, sich in ihrer Arbeitsmotivation von diesem zeitlichen Abstand beeinflussen zu lassen, desto geringer wird ihr Motivationsdruck, die Präsentation vorzubereiten, sein; desto größer ist also ihre Prokrastinationstendenz.

\subsection{Empirische Befunde}

Erwartung. Der Erwartungsaspekt des Modells findet sich im Konzept der Selbstwirksamkeitserwartung (engl.: perceived self-efficacy) wieder, das ursprünglich von Albert Bandura postuliert wurde und seit den 1970er-Jahren Einzug in die psychologische Forschung genommen hat. Die Selbstwirksamkeitserwartung eines Menschen bezeichnet seine Erwartung, aufgrund eigener Kompetenzen intendierte Handlungen erfolgreich ausführen und somit die erwünschten Handlungsergebnisse erzielen zu können. Personen, die glauben, auch in schwierigen Situationen selbstständig handeln und damit die angestrebten Wirkungen erreichen zu können, haben eine hohe Selbstwirksamkeitserwartung. In unserem Beispiel hätte eine Person, die glaubt, bei entsprechender Vorbereitung eine positiv bewertete Präsentation halten zu können, eine hohe Selbstwirksamkeitserwartung. Jemand, der daran zweifelt, ob er dies auch bei gründlicher Vorbereitung schafft, wäre dagegen wenig selbstwirksam. In der Tat gibt es konsistente empirische Befunde, die zeigen, dass eine höhere Selbstwirksamkeitserwartung mit geringerer Prokrastinationstendenz einhergeht. In einer Meta-Analyse von mehr als 200 Publikationen fand Steel (2007) beispielsweise eine mittlere Korrelation von $-0,38$.

Wert. Auch für die postulierte Rolle des Wertes der Verhaltenskonsequenz gibt es unterstützende empirische Befunde. Je aversiver (mühsamer, langweiliger, beängstigender etc.) eine Aufgabe ist, desto höher ist die Prokrastinationstendenz, besonders bei geringer Gewissenhaftigkeit. Je leistungsmotivierter eine Person dagegen ist (je höher also der subjektive Wert von Handlungserfolg), desto weniger neigt sie zur Prokrastination. Umgekehrt ist Anfälligkeit für Langeweile (die mit einer höheren Aufgabenaversion einhergeht) mit höherer Prokrastinationstendenz assoziiert. In der Meta-Analyse von Steel (2007) waren die mittleren Korrelationen für diese Zusammenhänge von Verhaltenskonsequenz und Prokrastination in der Größenordnung von um die 0,40 .

Belohnungsfrist. Konsistent mit der postulierten Rolle der Belohnungsfrist zeigen Studien, dass Prokrastination mit zunehmender Nähe zum Fristende (der Deadline) 
abnimmt (z. B. Howell und Watson 2007). Auch zeigt sich, dass organisierte Menschen eine geringere Prokrastinationstendenz aufweisen. Was tun nämlich organisierte Personen? Sie teilen ein langfristiges Ziel in kurzfristige Zwischenziele ein und verkürzen somit die Belohnungsfristen.

Fristsensitivität. Übereinstimmend mit der Annahme zur Rolle der Fristsensitivität schließlich zeigen Studienergebnisse, dass ablenkbarere und impulsive Personen eine höhere Prokrastinationstendenz aufweisen, während eine bessere Selbstkontrolle (also eine bessere Fähigkeit, Versuchungen widerstehen zu können) mit geringerer Prokrastinationstendenz einhergeht. Insbesondere der letztere Zusammenhang ist mit einer mittleren Korrelation von -0,58 in der Meta-Analyse von Steel (2007) als substantiell zu bewerten.

\section{Prokrastination als Coaching-Anliegen: Was tun?}

Wie lassen sich diese Einsichten nun im Coaching-Alltag nutzen? Hier sind zwei grundlegende Szenarien zu unterscheiden: Zum einen mag sich im Coaching herausstellen, dass Prokrastination von Mitarbeitern und Kollegen zu einem thematisierten Anliegen wird. Zum anderen mag der Coachee seine eigene als problematisch erlebte Prokrastinationstendenz als Thema in das Coaching einbringen.

\subsection{Prokrastination bei Kollegen oder Mitarbeitern des Coachees}

Dem Coach sollte bewusst sein, dass eine Veränderung der Prokrastinationstendenz dritter Personen, die nicht selbst in die Beratung involviert sind, kein geeignetes Coaching-Anliegen ist. Er oder sie sollte daher stets darauf drängen, dass der Coachee sein Anliegen so formuliert, dass ihn selbst betreffende Aspekte der Problemsituation adressiert werden, beispielsweise der Wunsch nach einem besseren Verständnis der Situation oder der Erarbeitung oder Veränderung eigener Handlungsstrategien zur Situationsbewältigung. In einem solchen Fall kann der Coach die Temporal Motivation Theory im Sinne der Psychoedukation vorstellen und als Explorations-Richtlinie nutzen, um den Coachee beim Erwerb eines besseren Verständnisses der problematischen Situation und der wirksamen Einflussfaktoren zu unterstützen. Ein solches Verständnis wiederum ist die Voraussetzung für die Entwicklung oder Veränderung von Strategien zum Umgang mit Prokrastination bei Kollegen oder Mitarbeitern.

\subsection{Prokrastination beim Coachee}

Auch wenn der Coachee die Reduktion seiner eigenen Prokrastinationstendenz in das Coaching einbringt, bietet sich die Temporal Motivation Theory als psychoedukative Maßnahme und Explorationsleitfaden an, um zu einem besseren Verständnis der Situation und insbesondere der wirksamen Einflussfaktoren zu gelangen. Sind diese herausgearbeitet, können konkrete Coachingmaßnahmen ausgewählt werden. Einige Möglichkeiten werden im Folgenden skizziert, wiederum anhand der ver- 
schiedenen Einflussfaktoren des Modells organisiert (für einen vertieften Überblick zu Interventionsmöglichkeiten vgl. Rozental und Carlbring 2014).

Erwartung. Wurde im Verlauf des Coachings herausgearbeitet, dass eine mangelnde Selbstwirksamkeitserwartung zur Prokrastinationstendenz beiträgt, sind Maßnahmen zur Steigerung von Selbstwirksamkeit indiziert. Es gibt einige Evidenz, dass Modell-Lernen anhand der Beobachtung von Erfolgserlebnissen anderer, denen sich der Coachee ähnlich fühlt, zur Erhöhung der Selbstwirksamkeitserwartung beitragen kann. Dies sollte jedoch nur ein erster Schritt sein, denn grundlegend am wirksamsten im Hinblick auf die Erhöhung der Selbstwirksamkeitserwartung sind eigene Kompetenz-Erfahrungen. Hilfreich ist zum Beispiel, sich relevanten, jedoch zunehmend schwierigeren Herausforderungen zu stellen, um so korrektives Feedback und Verstärkung zu erhalten. Auch die Arbeit an dysfunktionalen habituellen Kognitionen des Coachees - etwa Perfektionismus, Sorgen oder Versagensängste - kann in einem solchen Fall angezeigt sein. Je nach Einzelfall kann auch die Adressierung von Organisationsprozessen indiziert sein, beispielsweise anhand der folgenden Fragen: Sind die Zeitpläne und Abläufe so organisiert, dass Erfolge überhaupt möglich sind? Sind Fallback-Szenarien für Notfälle vorgesehen? Wenn nicht, was sollte wie geändert werden?

Wert. Die Arbeit mit der Temporal Motivation Theory im Coaching mag auch deutlich machen, dass ein geringer Wert der Handlung oder der Handlungskonsequenz - beispielsweise Langeweile oder Zweifel am Sinn der eigenen Arbeit zur Prokrastinationstendenz beiträgt. Hier kann der Coachee angeregt werden, über Möglichkeiten zur Erhöhung der Attraktivität der zu erledigenden Arbeiten nachzudenken. Ideal sind herausfordernde Aufgaben, die dennoch erfüllbar sind. Hierbei können das Formulieren von erreichbaren und attraktiven Zwischenzielen hilfreich sein oder auch das sogenannte Fusing, das Zusammenführen unterschiedlicher Interessen in derselben Handlung. Gemeint ist die Verbindung des Notwendigen mit dem Angenehmen - etwa das Bearbeiten unangenehmer Aufgaben in einem attraktiven Setting (z. B. auf einem Arbeitsgruppen-Retreat oder gemeinsam mit einer inspirierenden Gruppe von Kollegen). Scheint dem Coachee eine Erhöhung des Wertes des eigentlich in Frage stehenden Verhaltens nicht möglich, kann auch über die Möglichkeit externer Motivationen, beispielsweise das Sich-Selbst-Belohnen des Coachees bei fristgerechter Handlungsausführung nachgedacht werden. Je nach Einzelfall mag auch eine relativierende Einbettung in das übergeordnete Wertesystem des Coachees hilfreich sein. Hier geht es darum, die Relevanz des abgewerteten Verhaltens im Hinblick auf die übergeordneten Ziele und Werte (z. B. finanzielle Absicherung, WorkLife-Balance) zu explorieren. Wenn solche Wert-Fragen im Coaching zu Tage treten, sollte der Coach immer auf die Möglichkeit gefasst sein, dass der Coachee sein Coaching-Anliegen umdefiniert, da über die Bearbeitung des Prokrastinationsproblems grundlegende Wert- und Rollenkonflikte aufgedeckt wurden, die schließlich in die Frage einer möglichen Neuorientierung münden können.

Belohnungsfrist und Fristsensitivität. Arbeitet der Coachee heraus, dass seine hohe Sensitivität gegenüber langen Belohnungsfristen problematisch ist, dass er 
oder sie sich also schwer motivieren kann, vorausschauend auf langfristige Ziele hinzuarbeiten, sind Maßnahmen zum Zeitmanagement und zur Erhöhung der Selbstkontrolle angemessen. Zeitmanagement zielt auf eine Reduktion von Prokrastinationstendenz durch Senkung der Belohnungsfrist ab. Durch das vorausschauende Planen werden langfristiger Aufgabe in einzelne, konkret terminierte Arbeitsschritte unterteilt und die Belohnungsfrist so verkürzt. Hilfreich ist auch, möglicherweise auftretende Schwierigkeiten in der Umsetzung des Zeitplans zu antizipieren und Fallback-Szenarien für deren Auftreten mitzuplanen. In der Fachsprache wird dieses detaillierte vorausschauende Planen als Bildung von Implementations- und CopingIntentionen bezeichnet. Zahlreiche Forschungsbefunde weisen die Wirksamkeit dieser Maßnahmen im Hinblick auf eine erhöhte Persistenz in der Zielverfolgung nach. Auch Interventionen zur Erhöhung der Selbstkontrolle sind bei Problemen mit langer Belohnungsfrist und hoher Fristsensitivität relevant. Hierzu gehört z. B. das Verbannen möglicher Versuchungen (z. B. Schließen des Browsers oder Mailprogramms), während stattdessen sichtbare Erinnerungen an das intendierte Verhalten aufgestellt werden (z. B. ein Zeitplan ausgedruckt und an die Wand gehängt wird). Hilfreich sind hier auch eindeutige Verknüpfungen von Kontext und Verhalten und das Automatisieren von Verhalten durch das Einführen fester Routinen (z. B. wenn bestimmte Aufgaben immer zu vorgegebenen Zeiten und in einer festgelegten Reihenfolge und an einem definierten Ort erlediget werden).

\section{Schlussfolgerung}

Prokrastination ist eine häufige Form des nicht zielgerichteten und nicht durch äußere Zwänge hervorgerufenen Aufschubs intendierter Handlungen und hat potenziell negative Auswirkungen auf die betroffene Person und die Organisationseinheit, in der sie tätig ist. Prokrastinationsrelevante Anliegen im Coaching können sowohl mit Prokrastination von Mitarbeitern und Kollegen im Zusammenhang stehen als auch eigene Prokrastinationstendenzen des Coachees betreffen. Die Temporal Motivation Theory ist ein in der aktuellen nicht-klinischen Prokrastinationsforschung viel beachtetes Modell, das im Einklang mit vielfältigen empirischen Befunden steht. Es bietet sich als theoretischer Rahmen für die Bearbeitung von Prokrastinations-Anliegen im Coaching an. Seine Annahmen können dem Coachee leicht vermittelt werden und so zu einem besseren Verständnis der problematischen Situation und möglicher Einflussfaktoren beitragen. Zudem kann die Theorie dem Coach als Explorationsleitfaden für eine gründliche Erkundung des internen und des externen Kontexts und die Auswahl der im jeweiligen Einzelfall indizierten Interventionsmethoden dienen.

Open access funding provided by Max Planck Institute for Human Development.

Open Access Dieser Artikel wird unter der Creative Commons Namensnennung 4.0 International Lizenz (http://creativecommons.org/licenses/by/4.0/deed.de) veröffentlicht, welche die Nutzung, Vervielfältigung, Bearbeitung, Verbreitung und Wiedergabe in jeglichem Medium und Format erlaubt, sofern Sie den/die ursprünglichen Autor(en) und die Quelle ordnungsgemäß nennen, einen Link zur Creative Commons Lizenz beifügen und angeben, ob Änderungen vorgenommen wurden. 


\section{Literatur}

Chu, A. H. C., \& Choi, J. N. (2005). Rethinking procrastination: Positive effects of "active" procrastination behavior on attitudes and performance. Journal of Social Psychology, 14, 245-264.

Howell, A. J., \& Watson, D.C. (2007). Procrastination: associations with achievement goal orientation and learning strategies. Personality and Individual Differences, 43, 167-178.

Klingsieck, K. B. (2013). Procrastination: when good things don't come to those who wait. European Psychologist, 18, 24-34.

Krause, K., \& Freund, A. M. (2013). How to beat procrastination: the role of goal focus. European Psychologist, 19, 132-144.

Rhodewalt, F., \& Vohs, K. D. (2005). Defensive strategies, motivation, and the self. In A. Elliot \& C. Dweck (Hrsg.), Handbook of competence and motivation (S. 548-565). New York: Guilford Press.

Rozental, A., \& Carlbring, P. (2014). Understanding and treating procrastination: a review of a common self-regulatory failure. Psychology, 5, 1488-1502.

Simpson, W.K., \& Pychyl, T. A. (2009). In search of the arousal procrastinator: investigating the relation between procrastination, arousal-based personality traits and beliefs about procrastination motivations. Personality and Individual Differences, 47, 906-911.

Sirois, F.M. (2007). ,I'll look after my health, later“: a replication and extension of the procrastination-health model with community-dwelling adults. Personality and Individual Differences, 43, 15-26.

Steel, P. (2007). The nature of procrastination: a meta-analytic and theoretical review of quintessential selfregulatory failure. Psychological Bulletin, 133, 65-94.

Steel, P., \& König, C. J. (2006). Integrating theories of motivation. Academy of Management Review, 31, 889-913.

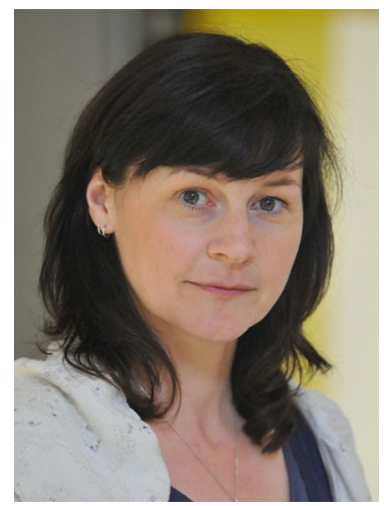

Dr. Michaela Riediger Leiterin der Heisenberg-Forschungsgruppe Sozioemotionale Entwicklung und Gesundheit im Lebensverlauf, Freie Universität Berlin und Max-Planck-Institut für Bildungsforschung, Berlin

(c) David Ausserhofer 